\title{
Impacto de la COVID-19 en el estilo de vida materno y vínculo de apego madre-recién nacido
}

Anghela Ramos Rodríguez ${ }^{1,2, a}$; Miryam Lora Loza*1,b; Juan Rodríguez Vega ${ }^{1, c}$; Jean Neil Hernández Angulo ${ }^{3, d}$; Hugo Eduardo Fernádez Cosavalente ${ }^{4,5, e}$; José Elías Cabrejo Paredes 5 ,f

\section{RESUMEN}

Objetivo: Determinar el impacto de la COVID-19 en la relación existente entre el estilo de vida y el vínculo del apego madre-recién nacido en un hospital de Perú.

Materiales y métodos: Se realizó una investigación explicativa, descriptiva-correlacional, de diseño transversal, con una población de 1020 puérperas y una muestra de 103 participantes. El muestreo fue estratificado por la presencia o no de COVID-19 en la población de estudio. Las variables fueron los estilos de vida (que incluye práctica de actividad física, calidad de vida, descanso y sueño, salud metal e integridad social) y el vínculo de apego madre-recién nacido. Se empleó la correlación de Spearman, análisis discriminante y la prueba de Wilcoxon.

Resultados: La población de estudio estuvo conformada por 51 puérperas con COVID-19 y 52 que no presentaron la enfermedad. Los estilos de vida fueron regulares en el 66,67 \% y 55,77 \%, respectivamente. En el primer grupo (con COVID-19), el vínculo de apego madre-recién nacido fue bueno $(64,71 \%)$, la práctica de actividad física resultó intensa (62,75\%) con una dieta saludable en el $84,31 \%$ de los casos, el descanso y sueño fueron adecuados (54,90\%), la salud mental y autoestima se calificaron como buenas en el $62,74 \%$, y la integración social resultó alta (68,63\%). En el segundo grupo (pacientes sin COVID-19), el vínculo de apego madre-recién nacido fue regular (55,76 \%), un 61,54 \% tuvo una práctica de actividad física moderada, la dieta fue saludable en el $69,23 \%$ de los casos, el descanso y el sueño fueron adecuados $(75,00 \%)$, la salud mental y autoestima tuvieron un nivel regular $(63,47 \%)$, y la integración social fue calificada como media $(67,31 \%)$.

Conclusiones: La COVID-19 tuvo un impacto significativo $(p<0,05)$ sobre la relación entre estilos de vida y el vínculo de apego materno con el recién nacido. El componente descanso y sueño fue un factor determinante del impacto de la COVID-19.

Palabras clave: Infecciones por Coronavirus; Estilo de vida; Recién nacido; Conducta materna; Afecto (Fuente: DeCS BIREME).

\section{Impact of COVID-19 on maternal lifestyle and mother-newborn bonding}

\section{ABSTRACT}

Objective: To determine the impact of COVID-19 on the relationship between lifestyle and mother-newborn bonding in a Peruvian hospital.

Materials and methods: An explanatory, descriptive-correlational, cross-sectional research was conducted with a population of 1,020 puerperal women and a sample of 103 participants. A stratified sampling was used to divide the study population into those with COVID-19 and those without COVID-19. The variables were lifestyles (including physical activity, quality of life, rest and sleep, mental health and social integration) and mother-newborn bonding. Spearman's rank correlation coefficient, discriminant analysis and Wilcoxon test were used.

Results: The study population consisted of 51 puerperal women with COVID-19 and 52 without COVID-19, out of whom $66.67 \%$ and $55.77 \%$ had a fair lifestyle, respectively. The first group (those with COVID-19) showed a good mother-newborn bonding (64.71\%), intense physical activity (62.75\%), healthy diet (84.31\%), adequate rest and sleep (54.90\%), good mental health and self-esteem (62.74\%), and high social integration (68.72\%). The second group (those without COVID-19) showed a fair mother-newborn bonding (55.76 \%), moderate physical activity (62.75\%), healthy diet (69.23\%), adequate rest and sleep (75.00\%), fair mental health and self-esteem (63.47 \%), and medium social integration (67.31\%).

Conclusions: COVID-19 had a significant impact $(p<0.05)$ on the relationship between lifestyles and mother-newborn bonding. Rest and sleep were key factors in determining the impact of COVID-19.

Keywords: Coronavirus infections; Life style; Infant, newborn; Maternal behavior; Affect (Source: MeSH NLM).

1 Universidad Cesar Vallejo. Trujillo, Perú.

2 Hospital Víctor Lazarte Echegaray- EsSalud. Trujillo, Perú.

3 Hospital Santa Gema. Trujillo, Perú.

4 Hospital Belén de Trujillo. Trujillo, Perú.

5 Universidad Nacional de Trujillo, Facultad de Medicina Escuela de Posgrado. Trujillo, Perú.

a Obstetra, Magister en Gestión de los Servicios de la Salud.

b Obstetra, Especialista en Emergencias Obstétricas, Doctora en Gestión y Planificación.

c Psicólogo, especialista en Biología y Química, Doctor en Ciencias de la Educación.

d Obstetra asistencial, Maestro en Gerencia Social.

e Médico especialista en Pediatría, Doctor en Medicina.

f Médico especialista en Medicina Familiar, Doctor en Medicina.

${ }^{*}$ Autor corresponsal.

Horiz Med (Lima) 2021; 21(2):e1354 


\section{INTRODUCCIÓN}

Uno de los problemas más relevantes generados a raíz de la aparición y expansión de la COVID-19 se ha centrado en los estilos de vida de las poblaciones en todo el mundo. Sus expresiones de confinamiento y aislamiento social y las llamadas distancias sociales no solo se introdujeron como una forma de comparar miedos, temores y pánicos, sino que estaban orientados, exclusivamente, a "evitar el contagio de la COVID-19", sin considerar que con ello se rompía la interacción básica entre las personas. Uno de los elementos concurrentes en este fenómeno médico-social fue el desconocimiento de la forma como se expandía realmente la enfermedad. Otro elemento fue la falta de ideas y de personas con habilidades y motivaciones positivas para enfrentar al COVID-19 como un problema médico-social y cultural (1).

Las mujeres con niños recién nacidos no tuvieron la atención necesaria para satisfacer sus necesidades de seguridad para mantener sus estilos de vida. La naturaleza misma de sus requerimientos básicos comenzó a cambiar y se destruyeron las prioridades a las que, culturalmente, estaban predispuestos la mujer y el recién nacido; por más que se buscó satisfacer las necesidades de actividad física, calidad de dieta, descanso, atención a su salud metal y autoestima, y de integridad social, ninguna sociedad en el mundo pudo hacerlo desde el inicio de la pandemia (2). Esta situación evidencia la relación que existe entre el nivel de estrés materno y el nivel de afectividad y de protección-apego entre madre-hijo ${ }^{(3)}$.

Los cambios de los estilos de vida estaban afectando la propia sobrevivencia del ser humano, lo que se manifestó en la desatención de sus necesidades básicas para el acceso al agua, alimento, aire, eliminación, descanso y sueño. Toda la vida de las personas debía girar en torno a "evitar el contagio del COVID-19" y, con ello, era imposible sostener una vida digna como la que se conocía y a la que se estaba acostumbrado. Las necesidades de confinamiento social obstruían las formas de obtener una alimentación saludable, una actividad física adecuada, un descanso y sueño reparadores, etc., sin que se avizore el final de esta situación y, lo que es peor, amenazando con extenderse tanto como para quebrar cualquier propósito sano de dar solución a los problemas médico-sociales que concurrieron o se desarrollaron a partir del COVID-19 ${ }^{(4)}$.

Ninguna de las políticas sanitarias en el mundo logró una atención adecuada de estos nuevos cambios en las necesidades existenciales de las personas, y quedó claro que la actividad física, la calidad de las dietas, el descanso y sueño, la salud mental y la integridad social, al igual que la afectividad, la protección y protección-apego materno estaban siendo afectados de manera directa. Países como Estados Unidos de Norteamérica, Alemania,
Canadá, Japón, entre otros, observaron con mucha preocupación este fenómeno, pero no le dieron ningún tipo de importancia porque el mayor porcentaje de sus mujeres gestantes estaban protegidas enteramente por los servicios de seguridad privados. Sin embargo, en países en vías de desarrollo de África, Asia, América Latina y el Caribe, más del $90 \%$ de las mujeres con sus recién nacidos vieron interrumpidos todo tipo de servicio de salud y asistencia social ${ }^{(5)}$.

En Perú, a pesar de que se trabajaron de inmediato medidas on-line para restablecer las consultas, interconsultas y los procesos de atención al binomio madre y niño, ninguna tuvo el impacto que se esperaba, es más, muy pocas personas siguieron las indicaciones para mejorar las prácticas de actividad física, la calidad de la dieta, el descanso y sueño, lo que llevó a que las estructuras mentales y la autoestima se dañaran. El impacto fue tan intenso que las personas se vieron fuera de su ambiente de interacción social natural y se les obligó a pensar solamente en el cuidado contra la COVID-19. Esto hizo que el objetivo familiar y social de las madres con recién nacidos vivos sea buscar refugio fuera del hogar para sentirse un poco seguras socialmente. Ya no era el ambiente familiar aquel que se conocía ni eran las mismas situaciones no familiares las que se encargaban de hacer trascender los estilos de vida para brindar afecto, protección y afectividad, protección-apego materna. Se recurrió así al uso de amuletos, talismanes u otros objetos mágico-religiosos para sentir protección frente al COVID-19 para salir a trabajar cada día, al no tener ninguna posibilidad de generar ingresos para garantizar la subsistencia e integridad de la familia. El COVID-19, poco a poco, fue dominando las necesidades de amor y pertenencia que se convirtieron en necesidades muy básicas e insignificantes. La sociedad conoce la gravedad que implica que los lactantes carezcan de amor y afecto: ellos no se desarrollan bien, por más que sus necesidades fisiológicas y de seguridad sean satisfechas. Es decir, los niños y jóvenes del futuro en nuestro país serán adultos carentes de amor y afecto que terminarán consumiendo drogas o implicados en actividades delictivas. A pesar de que la cercanía física entre personas podría haberle unido más, el efecto es contrario y muy negativo para la sociedad ${ }^{(6)}$. Estos fenómenos médico-sociales afectan directamente la salud mental de las personas, sus lazos familiares y con otras personas, pues se perdió una oportunidad para compartir amor y pertenencia, y se pasó a un abrupto estado de estar cerca y no poder dar amor ni sentido a la vida ${ }^{(7)}$.

El confinamiento social y la pérdida del trabajo, el menoscabo del valor social, del papel materno, de la dignidad individual y familiar de la mujer con su recién nacido no solo es comparable con lo que está ocurriendo con personas no "aseguradas", sino que todas ellas sienten que han sido degradadas socialmente, y esto 
genera sentimientos de desadaptación a su nueva realidad, frustraciones, enajenaciones $\mathrm{y}$, como resultado, desesperanza. Las madres con recién nacidos soportan preocupaciones que las alejan de su capacidad de brindar afecto, protección y afectividad: protección-apego materno. Se encuentran en medio de necesidades más apremiantes para la subsistencia individual y familiar. Jamás han sido tan afectadas en su potencial para logar sus objetivos de vida. Desde dicha perspectiva, se planteó como objetivo de esta investigación determinar el impacto del COVID-19 sobre la relación entre estilos de vida y el vínculo de apego madre-recién nacido en el Hospital III, EsSalud, Víctor Lazarte Echegaray.

\section{MATERIALES Y MÉTODOS}

\section{Diseño y población de estudio}

La investigación fue explicativa, descriptiva y correlacional de diseño transversal. La población estuvo conformada por 1020 puérperas atendidas en los servicios de emergencias obstétricas del Hospital III EsSalud Víctor Lazarte Echegaray de Trujillo. De ellas, 506 tuvieron diagnostico positivo de COVID-19 y 514 no presentaron la enfermedad. La muestra fue de 103: 51 con diagnostico positivo de COVID-19 y 52 puérperas sin COVID-19. Se aplicó un muestreo aleatorio simple y estratificado por la presencia o no de COVID-19 en la población de estudio. La muestra fue seleccionada de acuerdo a criterios de inclusión (puérperas atenidas en los servicios de emergencias obstétricas que participaron voluntariamente y en forma informada del Hospital III EsSalud Víctor Lazarte Echegaray) y criterios de exclusión (puérperas atendidas en el servicio de emergencia que presentaban problemas psicológicos o psiquiátricos, y las que no desearon participar del estudio).

\section{Variables y mediciones}

Las variables de la investigación fueron las siguientes: el estilo de vida (descrito por los indicadores: práctica de actividad física, calidad de vida, descanso y sueño, salud metal e integridad social) y la variable vínculo de apego madre-recién nacido. Estas variables fueron aplicadas a través de una encuesta con abordaje de persona a persona por vía telefónica. Los instrumentos se obtuvieron de la Encuesta Nacional de Salud del Instituto Nacional de
Estadística de España (2015), que articula los Cuestionarios de calidad de Vida (DEFA: Descanso, Ejercicio Físico y Alimentación), de Salud General (GHQ-12: Cuestionario de salud general, Goldberg y Williams, 1988), la Escala de Expectativas Específicas (BEEE: Batería de Escalas de Expectativas Específicas de Palenzuela, 2014) y la Escala de Afectividad, Protección y Afectividad, protección-apego Materno de la Organización Panamericana de la Salud (OPS).

\section{Análisis estadístico}

Para el análisis de correlación se empleó el coeficiente de correlación de Spearman R. Así mismo, se realizó un análisis discriminante lineal y se utilizó la prueba de Wilcoxon con signos para determinar las diferencias entre las relaciones de los grupos con y sin COVID-19.

\section{Consideraciones éticas}

Se consideraron los principios éticos del pronunciamiento de Helsinki y del informe Belmont como la autonomía, justicia, creatividad y aplicabilidad. Además, se garantiza el acceso a la información obtenida de cualquier análisis realizado por parte de las participantes del estudio. Asimismo, se respetaron los procedimientos éticos exigidos por el Comité de Ética de la Universidad César Vallejo y el empleo del paquete de información estadísticos tipográficos Turnitin para la prueba de que no hubo copia.

\section{RESULTADOS}

La tabla 1 muestra que los estilos de vida en puérperas con COVID-19 se califican como malos (1,96\%), regulares $(66,67 \%)$ y buenos $(31,37 \%)$; mientras que en puérperas sin COVID-19, malos $(19,23 \%)$, regulares $(55,77 \%)$ y buenos (25,00\%). El vínculo de apego madre-recién nacido en el grupo con COVID-19 es regular en el $35,29 \%$ y bueno en el $64,71 \%$; y en el grupo sin la enfermedad es regular en el $55,76 \%$ de las participantes y bueno en el $44,24 \%$. En ambos grupos, la correlación entre el estilo de vida y el vínculo de apego madre-recién nacido es significativa $(p<0,05)$. La diferencia entre ambos grupos no es significativa $(p<0,05)$, lo que sugiere que el COVID-19 tiene un impacto significativo sobre la relación entre los estilos de vida y el vínculo de apego madre-recién nacido (Tabla 1).

Tabla 1. Impacto de la COVID-19 sobre las relaciones entre el estilo de vida y el vínculo de apego madre-recién nacido en el Hospital III EsSalud Víctor Lazarte Echegaray

\begin{tabular}{|c|c|c|c|c|c|c|c|}
\hline \multirow[b]{3}{*}{ Estilos de vida } & & \multicolumn{6}{|c|}{ Vínculo de apego madre-recién nacido } \\
\hline & & \multicolumn{2}{|c|}{ Regular } & \multicolumn{2}{|c|}{ Bueno } & \multicolumn{2}{|c|}{ Total } \\
\hline & & N & $(\%)$ & N & $(\%)$ & N & $(\%)$ \\
\hline \multirow[t]{4}{*}{ Con COVID-19 } & Malos & 1 & 1,96 & 0 & 0,00 & 1 & 1,96 \\
\hline & Regulares & 15 & 29,41 & 19 & 37,26 & 34 & 66,67 \\
\hline & Buenos & 2 & 3,92 & 14 & 27,45 & 16 & 31,37 \\
\hline & Total & 18 & 35,29 & 33 & 64,71 & 51 & 100,00 \\
\hline
\end{tabular}




\begin{tabular}{|c|c|c|c|c|c|c|c|}
\hline \multirow[b]{3}{*}{ Estilos de vida } & & \multicolumn{6}{|c|}{ Vínculo de apego madre-recién nacido } \\
\hline & & \multicolumn{2}{|c|}{ Regular } & \multicolumn{2}{|c|}{ Bueno } & \multicolumn{2}{|c|}{ Total } \\
\hline & & N & $(\%)$ & N & $(\%)$ & N & (\%) \\
\hline \multirow[t]{4}{*}{ Sin COVID-19 } & Malos & 8 & 15,38 & 2 & 3,85 & 10 & 19,23 \\
\hline & Regulares & 17 & 32,69 & 12 & 23,08 & 29 & 55,77 \\
\hline & Buenos & 4 & 7,69 & 9 & 17,31 & 13 & 25,00 \\
\hline & Total & 29 & 55,76 & 23 & 44,24 & 52 & 100,00 \\
\hline
\end{tabular}

Fuente: elaboración propia

\begin{tabular}{|lcc|}
\hline \multicolumn{3}{|c|}{ Correlación por rangos de Spearman } \\
\hline Grupo & Coeficiente & Valor de $p$ \\
\hline Con COVID-19 & 0,348 & 0,012 \\
\hline Sin COVID-19 & 0,332 & 0,016 \\
\hline & Comparación de grupos (con y sin COVID-19) \\
\hline t de Student & \multicolumn{2}{c}{ Valor de $p$} \\
\hline 2,026 & 0,045 \\
\hline
\end{tabular}

En las puérperas con COVID-19 la práctica de actividad física fue calificada como suave $(1,96 \%)$, moderada $(35,29 \%)$ e intensa $(62,75 \%)$; mientras que en el grupo sin COVID-19 fue suave $(11,54 \%)$, moderada $(61,54 \%)$ e intensa $(26,92 \%)$. En relación a la calidad de la dieta, en el grupo con COVID-19 el 15,69 \% se define como no saludable y el $84,31 \%$, como saludable; en el grupo sin COVID-19 encontramos que el $30,77 \%$ fue no saludable y el $69,23 \%$ resultó saludable. Respecto al descanso y sueño, el $45,10 \%$ de las participantes con COVID-10 mostró un resultado no adecuado, y el 54,90\%, adecuado; para el grupo sin COVID-19, los resultados fueron no adecuado en $25,00 \%$ y adecuado en $75,00 \%$. Respecto a la salud mental y la autoestima encontramos las siguientes calificaciones: en el grupo con COVID-19, fue mala en el 1,96\%; regular, en el $35,30 \%$; y buena, en el $62,74 \%$. Mientras que en las puérperas sin COVID-19, fue mala en el 1,92\%; regular, en el $63,47 \%$; y buena, en el 34,61\%. La integridad social inherente a los estilos de vida en el grupo con COVID-19 resultó baja $(1,96 \%)$, media $(29,41 \%)$ y alta $(68,63 \%)$; y en el grupo $\sin$ COVID-19 es baja $(5,77 \%)$, media $(67,31 \%)$ y alta $(26,92 \%)$ (Tabla 2).

Tabla 2. Impacto de la COVID-19 sobre las relaciones entre indicadores de estilo de vida y el vínculo de apego madre-recién nacido en el Hospital III EsSalud Víctor Lazarte Echegaray

\begin{tabular}{|c|c|c|c|c|c|c|c|c|}
\hline \multirow{3}{*}{$\begin{array}{l}\text { Indicador del } \\
\text { estilo de vida }\end{array}$} & \multirow{3}{*}{ Grupo } & \multirow{3}{*}{$\begin{array}{l}\text { Nivel del } \\
\text { indicador }\end{array}$} & \multicolumn{6}{|c|}{ Vínculo de apego madre-recién nacido } \\
\hline & & & \multicolumn{2}{|c|}{ Regular } & \multicolumn{2}{|c|}{ Bueno } & \multicolumn{2}{|c|}{ Total } \\
\hline & & & N & $(\%)$ & N & $(\%)$ & N & $(\%)$ \\
\hline & & Suave & 1 & 1,96 & 0 & 0,0 & 1 & 1,96 \\
\hline \multirow[t]{8}{*}{ Práctica de actividad física } & Con COVID-19 & Moderada & 15 & 29,41 & 3 & 5,88 & 18 & 35,29 \\
\hline & & Intensa & 2 & 3,92 & 30 & 58,83 & 32 & 62,75 \\
\hline & & Total & 18 & 35,29 & 33 & 64,71 & 51 & 100,00 \\
\hline & & Suave & 1 & 1,92 & 5 & 9,62 & 6 & 11,54 \\
\hline & Sin COVID-19 & Moderada & 25 & 48,08 & 7 & 13,46 & 32 & 61,54 \\
\hline & & Intensa & 3 & 5,77 & 11 & 21,15 & 14 & 26,92 \\
\hline & & Total & 29 & 55,77 & 23 & 44,23 & 52 & 100,00 \\
\hline & & No saludable & 2 & 3,92 & 6 & 11,77 & 8 & 15,69 \\
\hline Calidad de la dieta & Con COVID-19 & Saludable & 16 & 31,37 & 27 & 52,94 & 43 & 84,31 \\
\hline
\end{tabular}




\begin{tabular}{|c|c|c|c|c|c|c|c|c|}
\hline \multirow{3}{*}{$\begin{array}{l}\text { Indicador del } \\
\text { estilo de vida }\end{array}$} & \multirow{3}{*}{ Grupo } & \multirow{3}{*}{$\begin{array}{l}\text { Nivel del } \\
\text { indicador }\end{array}$} & \multicolumn{6}{|c|}{ Vínculo de apego madre-recién nacido } \\
\hline & & & \multicolumn{2}{|c|}{ Regular } & \multicolumn{2}{|c|}{ Bueno } & \multicolumn{2}{|c|}{ Total } \\
\hline & & & N & $(\%)$ & N & $(\%)$ & N & $(\%)$ \\
\hline & & Total & 18 & 35,29 & 33 & 64,71 & 51 & 100,00 \\
\hline & & No saludable & 9 & 17,31 & 7 & 13,46 & 16 & 30,77 \\
\hline & Sin COVID-19 & Saludable & 20 & 38,46 & 16 & 30,77 & 36 & 69,23 \\
\hline & & Total & 29 & 55,77 & 23 & 44,23 & 52 & 100,00 \\
\hline & & No adecuado & 0 & 0,0 & 23 & 45,10 & 23 & 45,10 \\
\hline \multirow[t]{6}{*}{ Descanso y sueño } & Con COVID-19 & Adecuado & 18 & 35,29 & 10 & 19,61 & 28 & 54,90 \\
\hline & & Total & 18 & 35,29 & 33 & 64,71 & 51 & 100,00 \\
\hline & & No adecuado & 1 & 1,92 & 12 & 23,08 & 13 & 25,00 \\
\hline & Sin COVID-19 & Adecuado & 28 & 53,85 & 11 & 21,15 & 39 & 75,00 \\
\hline & & Total & 29 & 55,77 & 23 & 44,23 & 52 & 100,00 \\
\hline & & Mala & 1 & 1,96 & 0 & 0,00 & 1 & 1,96 \\
\hline \multirow[t]{8}{*}{ Salud mental y autoestima } & Con COVID-19 & Regular & 10 & 19,61 & 8 & 15,69 & 18 & 35,30 \\
\hline & & Buena & 7 & 13,73 & 25 & 49,01 & 32 & 62,74 \\
\hline & & Total & 18 & 35,30 & 33 & 64,70 & 51 & 100,00 \\
\hline & & Mala & 1 & 1,92 & 0 & 0,00 & 1 & 1,92 \\
\hline & Sin COVID-19 & Regular & 24 & 46,16 & 9 & 17,31 & 33 & 63,47 \\
\hline & & Buena & 4 & 7,69 & 14 & 26,92 & 18 & 34,61 \\
\hline & & Total & 29 & 55,77 & 23 & 44,23 & 52 & 100,00 \\
\hline & & Baja & 1 & 1,96 & 0 & 0,0 & 1 & 1,96 \\
\hline \multirow[t]{7}{*}{ Integridad social } & Con COVID-19 & Media & 10 & 19,61 & 5 & 9,80 & 15 & 29,41 \\
\hline & & Alta & 7 & 13,73 & 28 & 54,90 & 35 & 68,63 \\
\hline & & Total & 18 & 35,30 & 33 & 64,70 & 51 & 100,00 \\
\hline & & Baja & 3 & 5,77 & 0 & 0,00 & 3 & 5,77 \\
\hline & Sin COVID-19 & Media & 22 & 42,31 & 13 & 25,00 & 35 & 67,31 \\
\hline & & Alta & 4 & 7,69 & 10 & 19,23 & 14 & 26,92 \\
\hline & & Total & 29 & 55,77 & 23 & 44,23 & 52 & 100,00 \\
\hline
\end{tabular}

Fuente: elaboración propia.

La tabla 3 muestra que en puérperas con COVID-19 la correlación entre la práctica de actividad física, descanso y sueño, salud mental, autoestima e integridad social y el vínculo de apego madre-recién nacido es significativa $(p<0,05)$, mientras que en puérperas sin COVID-19 el descanso y sueño, la salud mental y autoestima y la integridad social están relacionados significativamente $(p<0,05)$ con el vínculo de apego madre-recién nacido.
Al comparar entre ambos grupos (con y sin COVID-19) se observa una diferencia significativa $(p<0,05)$ en relación a la práctica de actividad física, descanso y sueño, salud mental y autoestima e integridad social, lo que indica que la COVID-19 tiene un impacto significativo sobre la relación entre los estilos de vida y el vínculo de apego madre-recién nacido (Tabla 3). 
Tabla 3. El impacto del COVID-19 medido a través de la relación y comparación entre indicadores de estilo de vida y el vínculo de apego madre-recién nacido en el Hospital III EsSalud Víctor Lazarte Echegaray

\begin{tabular}{|c|c|c|c|}
\hline \multirow{2}{*}{$\begin{array}{l}\text { Indicador del estilo de vida } \\
\text { Práctica de actividad física }\end{array}$} & \multicolumn{3}{|c|}{ Correlación por rangos de Spearman } \\
\hline & Grupo & Coeficiente & Valor de $p$ \\
\hline & Con COVID-19 & 0,789 & 0,000 \\
\hline & Sin COVID-19 & 0,197 & 0,161 \\
\hline & \multicolumn{3}{|c|}{ Comparación de grupos (con y sin COVID-19) } \\
\hline & t de Student & \multicolumn{2}{|c|}{ Valor de $p$} \\
\hline & 4,035 & \multicolumn{2}{|l|}{0,000} \\
\hline \multirow[t]{7}{*}{ Calidad de la dieta } & \multicolumn{3}{|c|}{ Correlación por rangos de Spearman } \\
\hline & Grupo & Coeficiente & Valor de $p$ \\
\hline & Con COVID-19 & $-0,093$ & 0,517 \\
\hline & Sin COVID-19 & 0,006 & 0,964 \\
\hline & \multicolumn{3}{|c|}{ Comparación de grupos (con y sin COVID-19) } \\
\hline & t de Student & \multicolumn{2}{|c|}{ Valor de $p$} \\
\hline & 1,822 & \multicolumn{2}{|l|}{0,071} \\
\hline \multirow[t]{7}{*}{ Descanso y sueño } & \multicolumn{3}{|c|}{ Correlación por rangos de Spearman } \\
\hline & Grupo & Coeficiente & Valor de $p$ \\
\hline & Con COVID-19 & $-0,669$ & 0,000 \\
\hline & Sin COVID-19 & $-0,559$ & 0,000 \\
\hline & \multicolumn{3}{|c|}{ Comparación de grupos (con y sin COVID-19) } \\
\hline & t de Student & \multicolumn{2}{|l|}{ Valor de $p$} \\
\hline & $-2,167$ & \multicolumn{2}{|c|}{0,033} \\
\hline \multirow[t]{7}{*}{ Salud mental y autoestima } & \multicolumn{3}{|c|}{ Correlación por rangos de Spearman } \\
\hline & Grupo & Coeficiente & Valor de $p$ \\
\hline & Con COVID-19 & 0,376 & 0,007 \\
\hline & Sin COVID-19 & 0,497 & 0,000 \\
\hline & \multicolumn{3}{|c|}{ Comparación de grupos (con y sin COVID-19) } \\
\hline & t de Student & \multicolumn{2}{|l|}{ Valor de $p$} \\
\hline & 2,727 & \multicolumn{2}{|l|}{0,008} \\
\hline \multirow[t]{7}{*}{ Integridad social } & \multicolumn{3}{|c|}{ Correlación por rangos de Spearman } \\
\hline & Grupo & Coeficiente & Valor de $p$ \\
\hline & Con COVID-19 & 0,480 & 0,000 \\
\hline & Sin COVID-19 & 0,372 & 0,007 \\
\hline & \multicolumn{3}{|c|}{ Comparación de grupos (con y sin COVID-19) } \\
\hline & t de Student & \multicolumn{2}{|c|}{ Valor de $p$} \\
\hline & 4,386 & \multicolumn{2}{|l|}{0,000} \\
\hline
\end{tabular}

Fuente: elaboración propia.

En la tabla 4 se muestra el análisis discriminante lineal sobre datos relacionados con el impacto de la COVID-19 sobre la relación entre estilo de vida y el vínculo de apego madre-recién nacido en un hospital en Perú, donde se observa que el descanso y sueño y el vínculo de apego madre-recién nacido tienen mayor peso y determinan el impacto del COVID-19 sobre la relación entre estilos de vida y el vínculo de apego madre-recién nacido, permitiendo así discriminar a los dos grupos (con y sin COVID-19)(Tabla 4). 
Tabla 4. Factores que discriminan el impacto de la COVID-19 en la relación entre estilo de vida y el vínculo de apego madre-recién nacido en el Hospital III EsSalud Víctor Lazarte Echegaray

\begin{tabular}{|lr|}
\hline \multicolumn{2}{|c}{ Función discriminante lineal } \\
Factores & $\begin{array}{r}\text { Coeficientes } \\
\text { estandarizados }\end{array}$ \\
\hline Practica y actividad física & 5,992 \\
\hline Calidad de dieta & 6,796 \\
\hline Descanso y sueño & 22,115 \\
\hline Salud mental y autoestima & 4,426 \\
\hline Integridad social & 4,456 \\
\hline Gestión del apego madre-recién nacido & 17,310 \\
\hline Nivel general estilos de vida & $-0,798$ \\
\hline N = 103 & \\
\hline Proporción de clasificación correcta $=0,748$ & \\
\hline
\end{tabular}

\section{DISCUSIÓN}

Debido a que ninguna de las puérperas (con o sin COVID-19) fue capaz de adaptarse a la situación de manera paulatina y positiva, es probable que, en un primer momento de la pandemia, los procesos evolutivos del comportamiento las hicieron sentirse algo inseguras, sin percatarse de cuánto se había afectado su vida personal, familiar y social ${ }^{(8)}$. Debido al confinamiento social obligatorio, solo mostraron respuestas intuitivas sin tomar en cuenta lo que ocurría con sus vidas en el aspecto social ${ }^{(9)}$. En general, los impactos del COVID-19 se ocultan y solo se pueden evidenciar por los cambios en los comportamientos concurrentes que aparecen en situaciones de la vida de las puérperas ${ }^{(6)}$.

El hecho de que las puérperas infectadas de COVID-19 no tengan que ser hospitalizadas estimula actitudes positivas y genera nuevas posibilidades que permiten brindar mayor protección y desarrollar una gestión más significativa del apego materno con el recién nacido ${ }^{(10)}$. El tema de la calidad de las dietas muestra resultados de correlación inversa (favorable a puérperas con COVID-19). Esto indica que sus estilos de vida como sus posibilidades de desarrollar una buena gestión del apego materno con el recién nacido se condicionaron por los hechos de la coyuntura médica y social del COVID-19 ${ }^{(11)}$. Es posible que esta situación esté ocasionada por el instinto de la mujer que trata de retomar la condición y apariencia física que tenía antes del embarazo, y también por sentir el temor y la inseguridad que el COVID-19 imprime a su vida ${ }^{(12)}$.

La mujer que da lactancia a su hijo necesita alimentarse más para producir la cantidad de leche adecuada para la alimentación de su recién nacido. De hecho, las madres en lactancia requieren más líquidos en sus dietas para proporcionar el agua que debe llevar la leche materna (13). Este tema todavía está en un plano especulativo y no es tratado en este estudio para lograr una buena articulación del análisis interpretativo de los resultados ${ }^{(14)}$.

La gran mayoría de las puérperas que perdieron su trabajo formal comenzaron nuevos emprendimientos que les dan una motivación distinta y se instala un razonamiento basado en enclaves cognitivos básicos, como respuestas a las amenazas o a los miedos, temores y pánicos ocasionados por los hechos que les ha tocado vivir y que ellas no buscaron ${ }^{(15)}$.

Es posible que el impacto del COVID-19 en las relaciones entre los estilos de vida y el vínculo de apego madre-recién nacido haya sido consecuencia de la manera como la mujer ha desarrollado sus alternativas frente a la naturaleza de los acontecimientos ${ }^{(16)}$. El impacto del COVID-19 en la vida de las puérperas se ha reducido, sobre todo, en el grupo que ha tenido la enfermedad; sin embargo, ha estado a la par con aquellas que no la han padecido porque, de igual forma, tuvieron que seguir el confinamiento social obligatorio (1).

La orientación de los comportamientos y actitudes de las madres de los recién nacidos pudo haber inducido a la construcción de comportamientos positivos y brindar esperanza, a pesar de un futuro incierto ${ }^{(17)}$. Los resultados del estudio advierten la inminencia de un evento para el cual solo se esperaba establecer el impacto de un fenómeno médico y social (COVID-19) sobre las relaciones entre los estilos de vida y el vínculo de apego madre-recién nacido de puérperas con y sin la enfermedad, pero que se transformó en la señal que ilustra la forma como la mujer puede manejarse en medio de dicha contingencia ${ }^{(18)}$. Si no hay afecto en el vínculo de apego madre-recién nacido, predomina la desconfianza, en virtud de la cual nace la desesperanza, y la creencia de que no se puede satisfacer sus necesidades y cumplir sus deseos ${ }^{(19)}$. Si predomina la confianza, los individuos, desde niños, verán al mundo como un lugar acogedor, predecible y no tendrán problemas 
para establecer relaciones interpersonales y sociales ${ }^{(20)}$.

Para concluir, en puérperas con y sin COVID-19, se evidenció una relación significativa entre el estilo de vida y el vínculo de apego madre-recién nacido, y se logró identificar que el descanso y sueño, así como la gestión del apego madre-recién nacido, son factores determinantes del impacto del COVID-19 en la relación entre el estilo de vida y el vínculo de apego madre-recién nacido, que permiten diferenciar la respuesta de los dos grupos (con y $\sin$ COVID-19).

Contribuciones de los autores: Todos los autores han participado en cada etapa del estudio: concepción, diseño, recolección de datos, obtención de resultados, análisis e interpretación de los datos, redacción del informe, revisión crítica del manuscrito y aprobación de versión final informe.

Fuentes de financiamiento: El estudio ha sido financiado por los autores

Conflicto de Interés: Los autores declaran no tener ningún conflicto de interés.

\section{REFERENCIAS BIBLIOGRÁFICAS}

1. Brooks SK, Webster RK, Smith LE, Woodland L, Wessely S, Greenberg N, et al. El impacto psicológico de la cuarentena y cómo reducirlo: revisión rápida de la evidencia. The Lancet. 2020; 395(10227): 912-20.

2. Committee on World Food Security, High Level Panel of Experts. Impact of COVID-19 on Food Security and Nutrition (FSN) [Internet]. Roma; 2020. Disponible en: http://www.fao.org/fileadmin/templates/cfs/ Docs1920/Chair/HLPE_English.pdf

3. Cancho Rodríguez LI. Estrés materno y vinculo de afectividad, protecciónapego entre madre-hijo prematuro en hospital regional docente de Trujillo: 2018 [Tesis]. Trujillo: Universidad Nacional de Trujillo. Facultad de Medicina Humana; 2019.

4. Organización Panamericana de la Salud. Enfermedad por el Coronavirus (COVID-19) [Internet]. OPS; 2020. Disponible en: https://www.paho. org/es/temas/coronavirus/enfermedad-por-coronavirus-covid-19.

5. Organización Mundial de la Salud. Preguntas y respuestas sobre COVID-19, embarazo, parto y lactancia [Internet]. OMS; 2020. Disponible en: https://www.who.int/newsroom/q-a-detail/q-a-on-covid-19pregnancy-childbirth-and-breastfeeding.

6. Organización Panamericana de la Salud. Indicadores Básicos 2019: Tendencias de la Salud en las Américas 2019 [Internet]. OPS; 2020. Disponible en: https://iris.paho.org/handle/10665.2/51543

7. Rodríguez-Morales AJ, Sánchez-Duque JA, Hernández BS, Pérez-Díaz CE, Villamil-Gómez WE, Méndez CA, et al. Preparación y control de la enfermedad por coronavirus 2019 (COVID-19) en América Latina. Acta Med Perú. 2020; 37(1): 3-7.

8. Schroeder J, Kardas M, Epley N. La voz humanizadora: el habla revela y el texto oculta una mente más reflexiva en medio del desacuerdo. Psychol Sci. 2017; 28: 1745-62.

9. Haslam , Jetten J, Cruwys T, Dingle G, Haslam SA. The New Psychology of Health: Unlocking the Social Cure [libro electrónico]. Routledge: British Psychology Society Textbook; 2018. Disponible en: https: / www. routledge.com/The-New-Psychology-of-Health-Unlocking-the-SocialCure/Haslam-Jetten-Cruwys-Dingle-Haslam/p/book/9781138123885
10. World Health Organization. Improving early childhood development: WHO Guideline [Internet]. WHO; 2020. Disponible en: https://www.who. int/publications-detail/improving-earlychildhood-development-whoguideline

11. Van J, Baicker k, Boggio P. Uso de la ciencia social y del comportamiento para respaldar la respuesta a la pandemia de COVID-19. Rev Británica Psicol Soc. 2020; 4: 202-89.

12. Organización de las Naciones Unidas para la Alimentación y la Agricultura, Objetivos de Desarrollo Sostenible, Comunidad de Estados Latinoamericanos y Caribeños. Seguridad alimentaria bajo la pandemia de COVID-19* [Internet]. 2020. Disponible en: http://www.fao.org/3/ ca8873es/CA8873ES.pdf

13. Galanakis CM. The Food Systems in the era of the Coronavirus (COVID-19) pandemic crisis. Foods. 2020; 9(4): 523-33.

14. United Nations. Policy Brief: The impact of COVID-19 on Food Security and Nutrition [Internet]. 2020. Disponible en: https://www.un.org/sites/un2. un.org/files/sg_policy_brief_on_covid_impact_on_food_security.pdf

15. Abrams J. The game theory of panic-buying - and how to reduce it [Internet]. 2020. Disponible en: http://theconversation.com/the-gametheory-of-panic-buying-and-how-to-reduce-it-134107

16. Crum AJ, Jamieson JP, Akinola M. Optimizing stress: an integrated intervention for regulating stress responses. Emotion. 2020; 20(1): 120-5.

17. Marchlewska M, Cichocka A, Łozowski F, Górska P, Winiewski M. En busca de un enemigo imaginario: el narcisismo colectivo católico y el respaldo de las creencias de conspiración de género. J Soc Psychol. 2019; 159: 766-79.

18. Teeny J, Siev J, Briñol P, Petty R. Una revisión y un marco conceptual para comprender los efectos de correspondencia personalizados en la persuasión. J Consum Psychol. 2020; 1: 23-206

19. Erikson EH. Teoría psicosocial. Las ocho crisis. [Disponible]. Disponible en: http://www. Elgotero/Arcrivos\%20PDF/Teor\%C3\%ADa\%20Psicosocial\%20 Las\%200cho\%20Crisis\%20Erik\%20H.\%20Erikson.pdf

20. Berlin LJ, Ziv Y, Amaya-Jackson L, Greenberg MT. Enhancing early attachments: theory, research, intervention, and policy. J Can Acad Chilg Adolesc Psychiatry. 2007; 16(1): 33-6.

\section{Correspondencia:}

Miryam Lora Loza

Dirección: Calle Los Laureles 474 Urb. California. Dist.

Víctor Larco. Trujillo, La Libertad.

Teléfono: 964257604

Correo electrónico: mlora@ucv.edu.pe

Recibido: 26 de enero de 2021

Evaluado: 10 de febrero de 2021

Aprobado: 22 de febrero de 2021

(c) La revista. Publicado por Universidad de San Martín de Porres, Perú. (c) $\mathbf{B r}^{\mathbf{B}}$ Licencia de Creative Commons Artículo en acceso abierto bajo términos de Licencia Creative Commons Atribución 4.0 Internacional. (http://creativecommons.org/licenses/by/4.0/)

\section{ORCID iDs}

Anghela Ramos Rodríguez [i] https://orcid.org/0000-0001-5099-1314 Miryam Lora Loza $\quad$ https://orcid.org/0000-0001-9821-8531 Jean Neil Hernández Angulo (1) https: / / orcid.org/0000-0002-6852-472X Hugo Eduardo Fernádez Cosavalente $\odot$ https: / / orcid.org/0000-0002-2657-8287 José Elías Cabrejo Paredes (i) https: / / orcid.org/0000-0002-7335-0541 Juan Rodríguez Vega 